\title{
Fixkombination reduziert Langzeitkomplikationen bei BPH
}

\begin{abstract}
— Seit Mitte Juni 2010 steht mit Duodart ${ }^{\circledR}$ ein neues Medikament zur Behandlung der moderaten bis schweren benignen Prostatahyperplasie (BPH) zur Verfügung. Grundlage für die Markteinführung der Fixkombination aus dem $\alpha$-Rezeptorenblocker Tamsulosin (0,4 mg) und dem $5 \alpha$-Reduktasehemmer (5-ARI) Dutasterid (0,5 mg) waren die positiven Ergebnisse der multizentrischen Langzeitstudie CombAT [Roehrborn CG et al. Eur Urol 2010; 57:123-131]. Demzufolge konnte die kombinierte Gabe der beiden Wirkstoffe die unangenehmen Symptome der Prostatavergrößerung und das Risiko für schwerwiegende Komplika-
\end{abstract}

tionen besser reduzieren als die jeweiligen Monotherapien alleine. So erlitten nur 4,2\% der betroffenen Männer innerhalb von vier Jahren einen akuten Harnverhalt. oder benötigten eine $\mathrm{BPH}$-bedingte Operation, gegenüber 11,9\% unter isolierter Tamsulosin-Gabe. „Dies entspricht einer relativen Risikoreduktion von 66\%", rechnete PD Dr. Frank König, Berlin, vor. Die muskelrelaxierende Wirkung des $\alpha$-Blockers habe zu einer raschen Symptomlinderung geführt, die durch die hemmende Wirkung des 5-ARI auf das Prostatawachstum langfristig über die vierjährige Studiendauer aufrechterhalten werden konnte.
Die aktuellen deutschen Leitlinien empfehlen die Fixkombination bereits initial bei moderater Symptomatik (ab IPSS-Wert > 7) und gegebenem Progressionsrisiko (Prostatavolumen > $30 \mathrm{ml}$ ). Wie Dr. Matthias Oelke, Hannover, berichtete, war die neue Kapsel im Allgemeinen gut verträglich; die Häufigkeit unerwünschter Ereignisse einschließlich Störungen der Potenz und Libido war mit den beiden Monotherapien vergleichbar. „Man muss frühzeitig in die Therapie einsteigen, um die präventiven Effekte auf das Prostatawachstum nutzen zu können“, resümierte der Urologe. utz

Pressegespräch „Ein entscheidender Schritt im BPH-Management“, München, 18. Mai 2010.

Veranstalter: GlaxoSmithKline, München

\section{Erste Zweitlinientherapie für Patienten mit kastrationsresistentem Prostatakarzinom}

_ Für Patienten mit metastasiertem, kastrationsresistentem Prostatakarzinom (mKRPC), die nach/unter Firstline-Therapie mit Docetaxel progredient sind, wird mit Cabazitaxel in Kombination mit Prednison künftig erstmals eine Zweitlinientherapie zur Verfügung stehen, für die ein Überlebensvorteil nachgewiesen ist.

Cabazitaxel, ein neuartiges Taxan, kann die Taxan-Resistenz der Tumorzellen durchbrechen und ist auch bei Zelllinien wirksam, die nicht oder nicht mehr auf derzeit verfügbare Taxane ansprechen, erklärte Dr. Götz Geiges,
Berlin. Die Substanz ist derzeit bei der EMA zur Zulassung als Zweitlinientherapie des mKRPC eingereicht und wurde bereits in Kombination mit Prednison mit einem beschleunigten Zulassungsverfahren im Juni 2010 von der FDA für die USA zugelassen. Cabazitaxel/Prednison (CbzP) hat in der randomisierten Phase-III-Studie TROPIC mit 755 mit Docetaxel-vorbehandelten mKRPC-Patienten eine signifikante Verbesserung des medianen Gesamtüberlebens im Vergleich zur Behandlung mit Mitoxantron/Prednison (MP) erzielt. Das relative Sterberisiko wurde im CbzP-Arm signifikant um 30\% reduziert ( $p<0,0001)$. Die mittlere Gesamtüberlebenszeit betrug 15,1 Monate vs. 12,7 Monate im MP-Arm. Der mittlere Überlebensvorteil war unabhängig von Alter und Allgemeinzustand der Patienten sowie der Vorbehandlung. Das Verträglichkeitsprofil der Therapie war vorhersehbar, so Geiges. Neutropenie wurde bei $81,7 \%$ der CbzP-Patienten und bei $58 \%$ der MP-Patienten beobachtet. Schwere (Grad 3-4) nicht hämatologische Nebenwirkungen unter CbzP waren Durchfall (6\%), Übelkeit $(1,9 \%)$ und Erbrechen $(1,9 \%)$.

djb

Fachpresseworkshop „Erste Expertise Prostata“, Eltville, 27. Juli 2010. Veranstalter:

Sanofi Aventis, Frankfurt am Main

\section{Spontanes Liebesleben mit einmal täglich Tadalafil}

— Seit zwei Jahren steht für Männer mit Erektiler Dysfunktion (ED) neben den kurz und länger wirksamen PDE-5-Inhibitoren die tägliche Medikation mit Tadalafil (Cialis ${ }^{\circledR}$ ) 5 mg zur Verfügung. Kürzlich hat die European Association of Urology (EAU) diese dritte Option nun als Alternative zur Bedarfsmedikation in ihre Guidelines aufgenommen. In Studien war Tadalafil $5 \mathrm{mg}$ einmal täglich laut Prof. Hartmut Porst, Hamburg, vergleichbar wirksam und verträglich wie die Bedarfstherapie mit 20 mg. Im Gegensatz zu letzterer erlaube die tägliche Gabe aber ein fast so spontanes Liebesleben wie bei nicht von ED betroffenen Männern. Das wurde in einer aktuellen Studie belegt [Hatzimouratidis K et al. Eur Urol 2010; 57: 804-814]. Erste Hinweise sprechen dafür, dass die Wirkung der einmal täglichen Gabe von 5 mg Tadalafil zudem über den Behandlungszeitraum hinaus anhält, so Porst. In einer aktuellen post hoc-Analyse [Porst et al., J Sex Med 2010; 7:414-44] wurde die Nachhaltigkeit der Tadalafilwirkung bei 136 Patienten analysiert, deren Erektionsfähigkeit gegenüber den Ausgangswerten unter Tadalafil deutlich gebessert war. Bei 63 Patienten (46,3\%) blieb die Erektionswirkung vier Wochen nach Ende der Behandlung verbessert, darunter hielten 38 (60,3\%) die Erektionsfunktion in derselben IIEF-EF-Kategorie wie unter der Therapie und 25 (39,7\%) blieben immerhin noch eine IIEF-EF-Kategorie über den Ausgangswerten.

ay

Expertengespräch „Flexibler und freier. Einmal täglich Tadalafil in der ED-Therapie", 28. Juli 2010, Frankfurt am Main. Veranstalter: Lilly, Bad Homburg 